\title{
Topological Method Studying the Influence of Substituents and Structural Factors on Dependence "Structure-Property" of Aniline and its Derivatives
}

Mustafa S. Salakhov*, Olga T. Grechkina, Fatime A. Mustafayeva and Balakishi T. Bagmanov Institute of Polymer Materials of Azerbaijan National Academy of Science 124 Samed Vurgun Str., Az 5004, Sumgait, Azerbaijan

Received 09.10.2015, received in revised form 24.11.2015, accepted 03.12.2015

The results of calculations of the modified theoretical-information indices taking into account electronegativity constants and radiuses of atoms for aniline and its derivatives have been firstly presented and the possibilities of their use for establishment of dependence between structure and b.p. and m.p. have been shown.

Keywords: aniline; o-, $m$-, $p$ - substituted aniline; the modified theoretical-information indice; structure; the correlation analysi;, melting point; boiling point, property.

DOI: $10.17516 / 1998-2836-2015-8-4-559-569$.

(c) Siberian Federal University. All rights reserved

* Corresponding author E-mail address: salahov_mustafa@mail.ru 


\title{
Топологический метод изучения влияния \\ заместителей и структурных факторов \\ на зависимость «структура-свойство» анилина \\ и его производных
}

\author{
М.С. Салахов, О.Т. Гречкина, \\ Ф.А. Мустафаева, Б.Т. Багманов \\ Институт полимерных материалов \\ Национальной академии наук Азербайджана \\ Азербайджан, аз 5004, г. Сумгаит, ул. С. Вургуна, 1241
}

Впервые приведены результаты расчетов модифицированных теоретико-информационных индексов $I C_{k}^{\prime}, T I C_{k}^{\prime}, S I C_{k}^{\prime}$ и CIC $C_{k}^{\prime}(k=0-2)$ с учетом констант электроотрицательности и радиусов атомов для анилина и его производных и показаны возможности их использования для установления зависимости между их структурой и $T_{\text {кип }} u T_{n л}$.

Ключевые слова: анилин, о-, м-, п-замещенные анилина, модифищчрованные теоретикоинформационные индексы, структура, температура плавления, температура кипения, свойство.

Изучение зависимостей свойств продуктов реакции диенового синтеза от структуры их молекул может дать ключ к решению проблемы прогнозирования свойств аддуктов. Для установления зависимости «структура-свойство» соединений в современной органической химии нередко используют методы, основанные на описании строения молекулы структурной формулой. Одним из таких методов является топологический подход, при котором учитывают лишь типы атомов и характер их связывания, но пренебрегают метрическими характеристиками молекулы. В настоящее время исследованы некоторые корреляционные зависимости физикохимических свойств и других констант циклических аддуктов реакции Дильса-Альдера от топологических индексов (ТИ) [1-3], но проблема прогнозирования свойств аддуктов с заданным набором свойств изучена недостаточно.

С нашей точки зрения, представляет особый интерес организация систематических исследований по изучению применения ТИ для установления зависимости «структура-свойство» и проведения направленного синтеза по реакции Дильса-Альдера. В наших работах по исследованию и анализу взаимосвязи физико-химических свойств аддуктов диенового синтеза от структуры их молекул, описываемых с помощью ТИ Винера (W), Рандича ( $\chi$ ), а также теоретикоинформационных индексов: информационного содержания графа относительно окрестности k-го порядка $\left(\mathrm{IC}_{\mathrm{k}}\right)$ в расчете на одну вершину, полного информационного содержания $\left(\mathrm{TIC}_{\mathrm{k}}\right)$, структурного информационного содержания $\left(\mathrm{SIC}_{\mathrm{k}}\right)$ и комплементарного информационного содержания $\left(\mathrm{CIC}_{\mathrm{k}}\right)$ [4], мы показали удовлетворительную возможность использования $\mathrm{TИ} \mathrm{IC}_{\mathrm{k}}, \mathrm{TIC}_{\mathrm{k}}, \mathrm{SIC}_{\mathrm{k}}$ для установления зависимости между структурой и свойствами диеновых аддуктов [5-8] и предпо- 
чтительность теоретико-информационных индексов относительно индексов Винера W и Рандича ${ }^{(1)} \chi$ в установленных линейных корреляциях [6]. Для реализации поставленной цели из всего многообразия ТИ нами были выбраны теоретико-информационные индексы, или индексы симметрии окрестности, имеющие то преимущество, что они учитывают не только ближайшие, но и отдаленные взаимодействия в молекуле, тем самым обеспечивают наиболее полное описание структуры молекулы. В рамках решения задачи по направленному синтезу с целью подтверждения полученных теоретических результатов экспериментальными данными нами синтезированы соединения с предварительно спрогнозированными свойствами [9-11]. Для некоторых органических соединений, в частности для алканов и соответствующих галогенуглеродов, а также для галогензамещенных анилина использование индексов $\mathrm{IC}_{\mathrm{k}}, \mathrm{TIC}_{\mathrm{k}}, \mathrm{SIC}_{\mathrm{k}}$ и $\mathrm{CIC}_{\mathrm{k}}$ для установления зависимости «структура-свойство» невозможно, так как при расчете теоретикоинформационных индексов для отнесения вершины к какому-либо классу эквивалентности учитываются типы атомов и характер их связывания в молекуле, но, тем не менее, для указанных соединений индексы будут иметь соответственно равные значения. Многие из существующих топологических индексов (ТИ) [12-14] зависят только от структуры молекулы, но не от ее состава, поэтому молекулы одинаковой структуры, но разного состава при таких расчетах будут иметь равные индексы. В связи с этим был предложен ряд индексов, учитывающих природу составляющих молекулу атомов, например индексы электроотрицательности [15-17], модифицированный индекс Винера [18].

Для преодоления вышеуказанного затруднения мы ввели коэффициент электроотрицательности и значение радиусов атомов в существующе теоретико-информационные индексы $\mathrm{IC}_{\mathrm{k}}, \mathrm{TIC}_{\mathrm{k}}, \mathrm{SIC}_{\mathrm{k}}, \mathrm{BIC}_{\mathrm{k}}$ и $\mathrm{CIC}_{\mathrm{k}}$, несколько изменив методику их расчета. Введение именно этих параметров обусловлено необходимостью качественного связывания ТИ со строением атомов отдельных химических элементов и более точного отражения природы связей в молекулярной системе. Как мы показали ранее на примере алканов $\mathrm{C}_{1}-\mathrm{C}_{13}$, фторуглеродов $\mathrm{C}_{1}-\mathrm{C}_{8}$ и моногалогензамещенных бутанов, данная методика позволяет получать различие в значениях индексов для углеводородов и их галогензамещенных, что дает возможность описывать с их помощью соединения такого типа и устанавливать зависимость между их структурой и физико-химическими свойствами $[19,20]$. На примере бромированных имидов циклических дикарбоновых кислот показана перспектива применения модифицированных теоретико-информационных индексов для циклических аддуктов в решении задачи «структура-свойство» [21].

Целью данной работы является изучение зависимости свойств производных анилина от топологии их молекул: исследование влияния различных заместителей и их положения на существование зависимости «структура - свойство», так как свойства конечных продуктов в основном определяются природой присутствующих в соединении функциональных групп. В данной работе впервые приведены результаты расчетов модифицированных теоретикоинформационных индексов $\mathrm{IC}_{\mathrm{k}}{ }^{\prime}, \mathrm{TIC}_{\mathrm{k}}{ }^{\prime}, \mathrm{SIC}_{\mathrm{k}}{ }^{\prime}$ и $\mathrm{CIC}_{\mathrm{k}}{ }^{\prime}$ с учетом констант электроотрицательности и радиусов атомов по формулам (1-4) для анилина и его производных (I-XXII) и показаны возможности их использования для установления зависимости между их структурой и $\mathrm{T}_{\text {пл }}$ и $\mathrm{T}_{\text {кип. }}$

$$
\begin{gathered}
I C_{k}^{\prime}=-\sum_{i=1}^{h} \frac{R_{i}}{E_{i}} \cdot p_{i} \cdot \log _{2} p_{i}, \quad p_{i}=\frac{n_{i}}{n} \\
-561-
\end{gathered}
$$




$$
\begin{aligned}
& T I C_{k}^{\prime}=n \cdot I C_{k}{ }^{\prime} ; \\
& S I C_{k}^{\prime}=\frac{I C_{k}^{\prime}}{\log _{2} n} ; \\
& C I C_{k}^{\prime}=\log _{2} n-I C_{k}^{\prime},
\end{aligned}
$$

где $n$ - число вершин графа, равное $n=\sum_{i} n_{i} ; n_{i}-$ количество вершин в подмножестве $i ; N$ - чис-

ло ребер графа; $E_{i}$ - коэффициент электроотрицательности атома; $R_{i}$ - радиус атома.

Выбор производных анилина в качестве объекта исследования обусловлен возможностью варьирования их свойств в зависимости от изменения электронного характера, месторасположения и пространственного фактора заместителей, а также широким применением их в синтезе органических соединений. Нами ранее было изучено влияние структурных факторов и природы растворителя в реакциях бромирования анилинов, особенности поведения о-, м-, п-замещенных анилинов в данной реакции [22], описан способ получения моно-(IV) (A), а также ди- и триброманилинов и выявлено, что результат бромирования зависит как от характера заместителя, так и от его положения в ароматическом ядре.

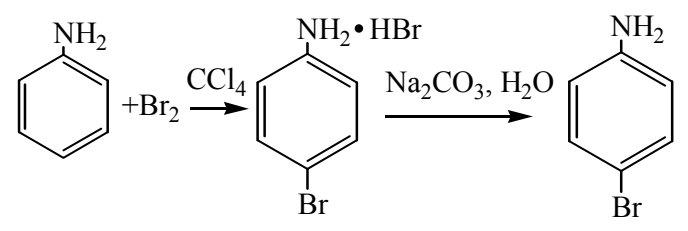

(I)

(IV)

A

Также исследовано окислительное бромирование анилина и его производных, содержащих в о-, м-, п-положении различные заместители $\left(\mathrm{CH}_{3}, \mathrm{Cl}, \mathrm{NO}_{2}, \mathrm{COOH}\right)$, и выявлено, что окислительное бромирование о- и п-замещенных анилинов отличается от бромирования м-замещенных производных и не зависит от электронной природы заместителей в фенильном кольце [23].

Соединения (II-IV), (V-VII), (VIII-X), (XI-XIII), (XIV-XVI), (XVII-XIX), (XX-XXII), являющихся о-, м-, п-изомерами соответствующих соединений, значения теоретико-информационных индексов $\mathrm{IC}_{\mathrm{k}}{ }^{\prime}, \mathrm{TIC}_{\mathrm{k}}{ }^{\prime}, \mathrm{CIC}_{\mathrm{k}}{ }^{\prime}$, и $\mathrm{SIC}_{\mathrm{k}}{ }_{\mathrm{k}}$ нулевого и первого порядков соответственно равны (табл. 1), из чего следует вывод о неспособности индексов указанных порядков участвовать в установлении корреляционных зависимостей «структура-свойство» для исследуемых соединений. Рассчитав теоретико-информационные индексы $\mathrm{IC}_{\mathrm{k}}{ }^{\prime}, \mathrm{TIC}_{\mathrm{k}}{ }^{\prime}, \mathrm{CIC}_{\mathrm{k}}{ }^{\prime}$, и $\mathrm{SIC}_{\mathrm{k}}{ }^{\prime}$ второго порядка, мы получили следующую закономерность для каждого из соединений исследуемого ряда: значения $\mathrm{IC}_{2}^{\prime}, \mathrm{TIC}_{2}^{\prime}$ и $\mathrm{SIC}_{2}^{\prime}$ при изменении положения заместителя увеличиваются следующим образом: п- $<$ o- $<$ M-.

Наименьшее значения указанных индексов именно в п-положении любого из заместителей является следствием симметричности графа, описывающего структуру соединения в этом положении (рис. 1), так как чем более симметричен граф, тем меньшим значением индекса $\mathrm{IC}_{\mathrm{k}}{ }^{\prime}$ 


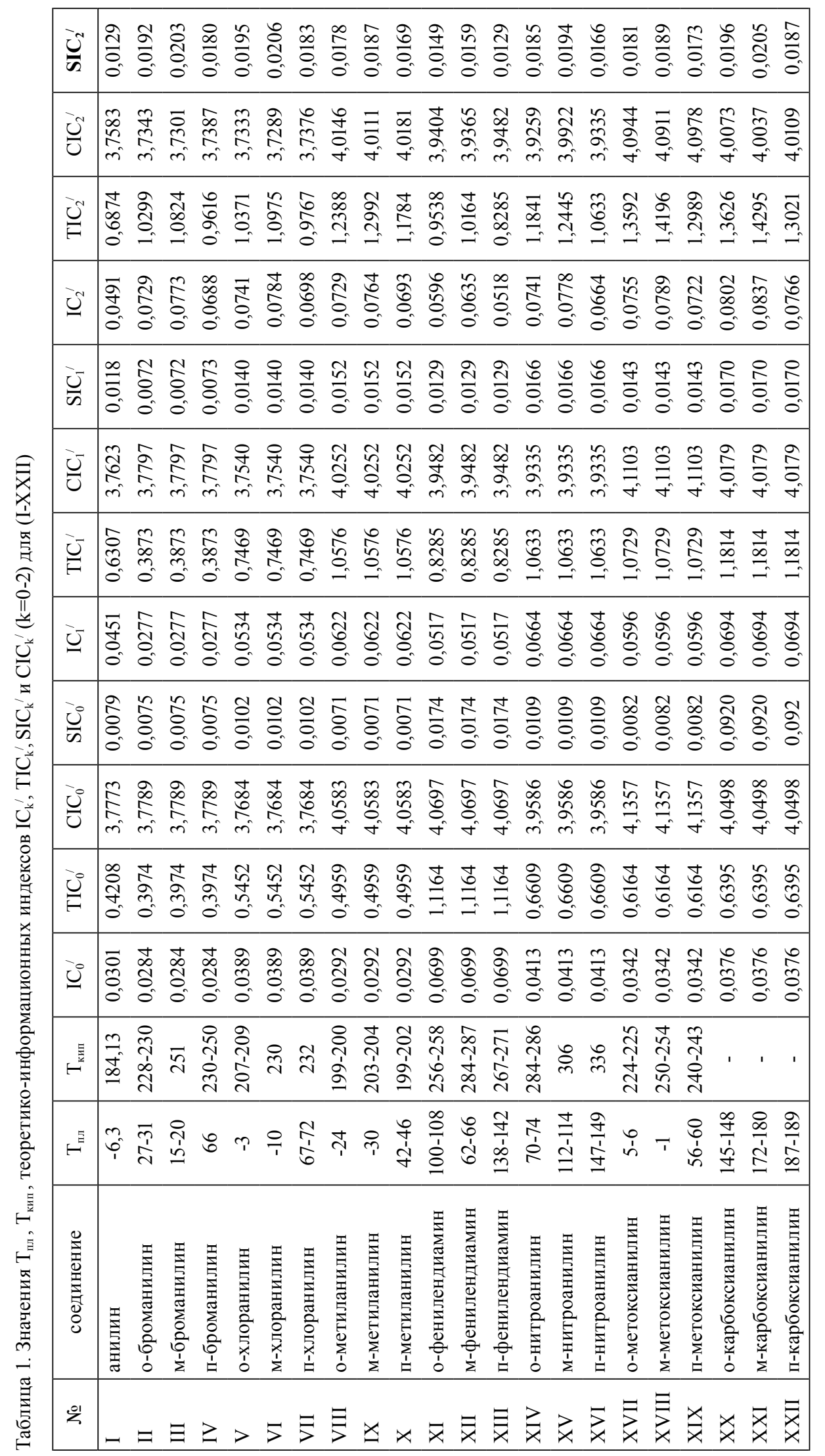




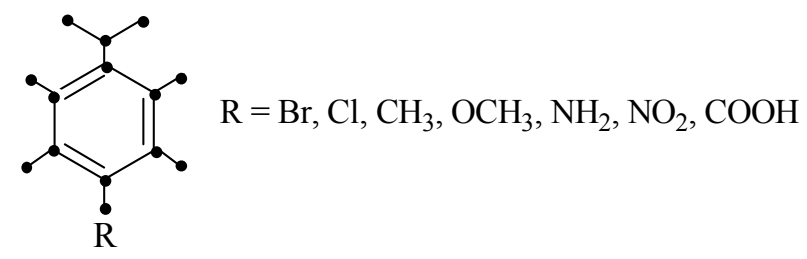

Рис. 1. Граф молекулы замещенного анилина в п-положении

обладает молекула, описываемая с помощью этого графа [4], следовательно, исходя из формул для расчета индексов $\mathrm{TIC}_{\mathrm{k}}{ }^{\prime}$ и $\mathrm{SIC}_{\mathrm{k}}{ }^{\prime}(2,3)$, индексы $\mathrm{TIC}_{2}^{\prime}$ и $\mathrm{SIC}_{2}^{\prime}$ также будут обладать наименьшим значением.

Как известно [24], электрофильная атака в ароматическом кольце анилина происходит по положению с максимальной электронной плотностью, т.е. в о- и п-положениях. Наибольшее значение указанных индексов именно в м-положении бензольного кольца анилина соответствует наименьшей электронной плотности и, как следствие, затруднению процесса замещения атома водорода в этом положении.

Использовав полученные нами значения модифицированных теоретико-информационных индексов (ТИ) второго порядка $\mathrm{IC}_{2}{ }^{\prime}, \mathrm{TIC}_{2}^{\prime}, \mathrm{CIC}_{2}{ }^{\prime}$, и $\mathrm{SIC}_{2}{ }^{\prime}$ и имеющиеся в литературе значения $\mathrm{T}_{\text {пл }}$ (табл. 1), мы установили существование линейных корреляционных зависимостей $\mathrm{f}(\mathrm{TИ})-\mathrm{T}_{\text {пл }}$ для о-, м-, п-броманилина (II-IV), описанных регрессионными уравнениями (5-9).

$$
\begin{array}{ll}
\text { Tnл }=-5376,3 \cdot I C_{2}^{\prime}+431,47 & \mathrm{R}^{2}=0,9052 \\
\text { Tnл }=-387,25 \cdot T I C_{2}^{\prime}+435,79 & \mathrm{R}^{2}=0,9536 \\
\text { Tnл }=5369,5 \cdot \text { CIC }_{2}^{\prime}-20013 & \mathrm{R}^{2}=0,9241 \\
\text { Tnл }=-20139 \cdot \text { SIC }_{2}^{\prime}+424,99 & \mathrm{R}^{2}=0,9301
\end{array}
$$

Для о-, м- и п-хлоранилинов (V-VII) и о-, м- и п-метиланилинов (VIII-X), как и для (II-IV), между модифицированными теоретико-информационными индексами второго порядка и $\mathrm{T}_{\text {пл }}$, $\mathrm{T}_{\text {кип }}$ существуют подобные линейные корреляционные зависимости (рис. 2).

Несмотря на то что наличие двух аминогрупп в фенилендиамине отличает его свойства от свойств моногалогенанилинов (II-IV, V-VII), для изомеров фенилендиамина (XI-XIII) также существуют корреляционные зависимости $\mathrm{f}\left(\mathrm{ТИ)}-\mathrm{T}_{\text {пл }}\right.$, примером которой служит уравнение (9). Как следует из близких значений коэффициентов $\mathrm{k}$ уравнений (6) и (9), прямые образуют примерно один и тот же угол наклона с положительным направлением оси $\mathrm{TIC}_{2}{ }^{\prime}$, что указывает на единый характер описанных этими уравнениями зависимостей.

$$
\operatorname{Tn} л=-371,75 \cdot T I C_{2}^{\prime}+448,14, \quad \mathrm{R}^{2}=0,9752 .
$$

Также п-фенилендиамин (XIII) с п-бром-(IV), п-хлор-(VII), п-метиланилинами(X) образует линейную корреляционную зависимость (10):

$$
\text { Tкun }=-193,82 \cdot T I C_{2}^{\prime}+427,41, \quad \mathrm{R}^{2}=0,9732 .
$$




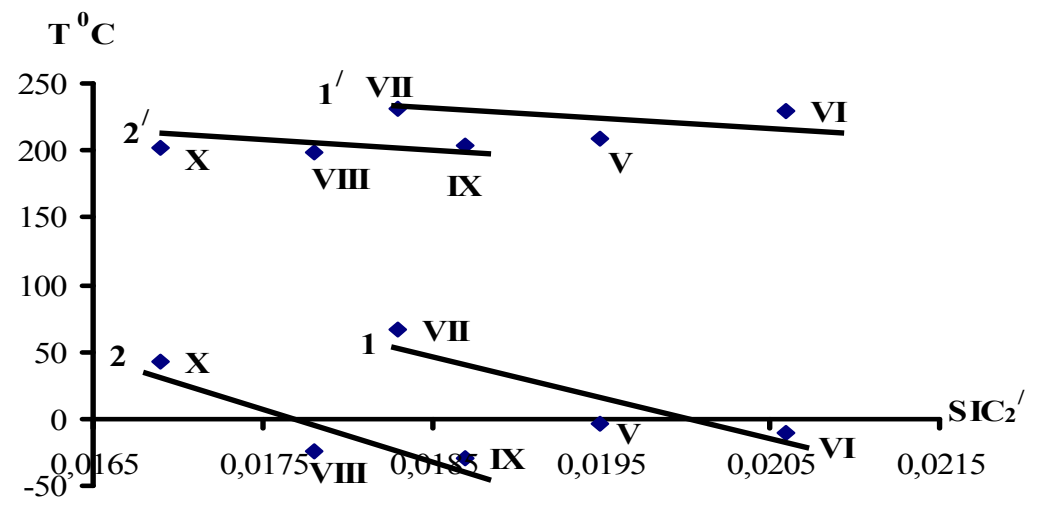

Рис. 2. Корреляционные зависимости $\mathrm{f}\left(\mathrm{SIC}_{2}{ }^{\prime}\right)-\mathrm{T}_{\text {пл }}$ (ряд 1 (V-VII), ряд 1'(VIII-X)) и f( $\left.\mathrm{SIC}_{2}{ }^{\prime}\right)-\mathrm{T}_{\text {кип }}$ (ряд 2 (V-VII), ряд 2/(VIII-X))

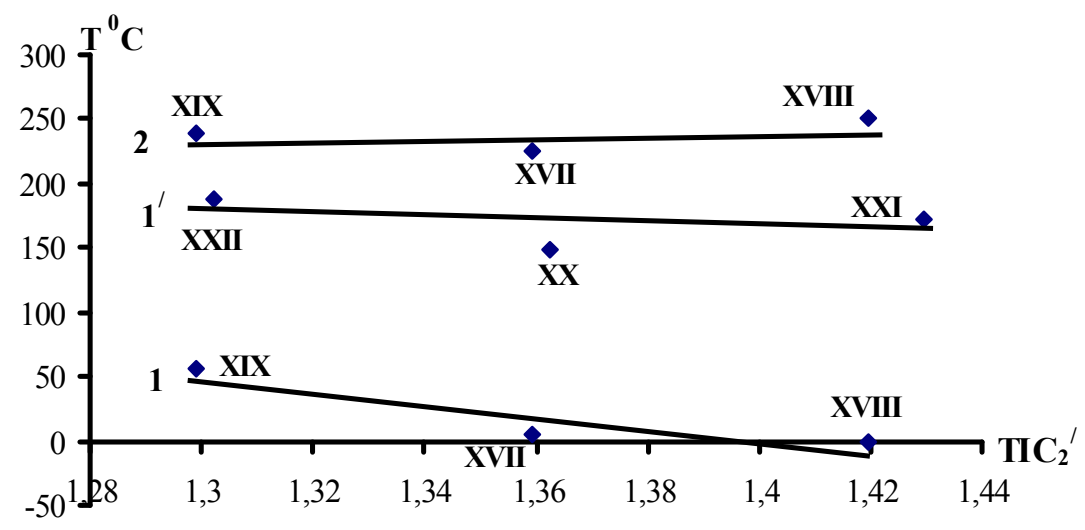

Рис. 3. Корреляционные зависимости $\mathrm{f}\left(\mathrm{TIC}_{2}{ }^{\prime}\right)-\mathrm{T}_{\text {пл }}$ (ряд 1 (XVII-XIX), ряд $\left.1^{\prime}(\mathrm{XX}-\mathrm{XXII})\right)$ и $\mathrm{f}\left(\mathrm{TIC}_{2}{ }^{\prime}\right)-\mathrm{T}_{\text {кип }}($ ряд $2(\mathrm{XVII}-\mathrm{XIX}))$

Метокси- (XVII-XIX) и карбоксианилины (XIX-XXI) также обладают подобными соотношениями между $\mathrm{T}_{\text {пл }}$ и индексами $\mathrm{IC}_{2} /, \mathrm{TIC}_{2}{ }^{\prime}, \mathrm{SIC}_{2}{ }^{\prime}$ и $\mathrm{CIC}_{2}{ }^{\prime}$ (рис. 3). Для соединений (XVII-XIX) также показано существование зависимости $\left(\mathrm{TIC}_{2}{ }^{\prime}\right)-\mathrm{T}_{\text {кип }}$ (рис. 3).

Для о-, м-, п-нитроанилина (XIV-XVI) получены антибатные зависимости $\mathrm{f}\left(\mathrm{TIC}_{2}{ }^{\prime}\right)-\mathrm{T}_{\text {пл }}$ и $\left(\mathrm{TIC}_{2}{ }^{\prime}\right)-\mathrm{T}_{\text {кип }}$ (рис. 4).

Несмотря на то, что соединения (II-IV), (V-VII) являются галоидзамещенными анилина, а соединения (VIII-X) и (XI-XIII) содержат соответственно метильную и карбоксильную группы, можно проследить наличие корреляционной зависимости между этими соединениями $\mathrm{f}\left(\mathrm{TIC}_{2}{ }^{\prime}\right)-\mathrm{T}_{\text {пл }}$ (рис. 5). Как и предполагалось, анилин (I) отклоняется от полученной зависимости, так как его молекула не имеет заместителей в о-, м- и п-положениях.

Установленные для вышеприведенных производных анилина (II-XXII) зависимости между модифицированными теоретико-информационными индексами и $\mathrm{T}_{\text {пл }}$ и $\mathrm{T}_{\text {кип }}$ позволяют предположить, что подобные соотношения между этими ТИ и физико-химическими свойствами могут существовать и для диеновых аддуктов, полученных на их основе, что даст возможность

$$
-565-
$$




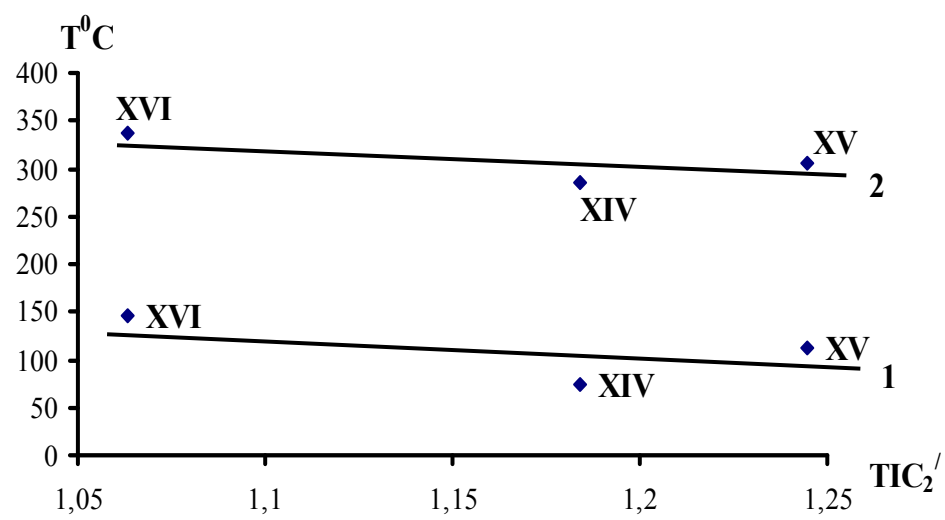

Рис. 4. Корреляционные зависимости $\mathrm{f}\left(\mathrm{TIC}_{2}{ }^{\prime}\right)-\mathrm{T}_{\text {пл }}$ (ряд 1) и $\mathrm{f}\left(\mathrm{TIC}_{2}{ }^{\prime}\right)-\mathrm{T}_{\text {кип }}$ (ряд 2) для о-, м-, п-нитроанилина (XIV-XVI)

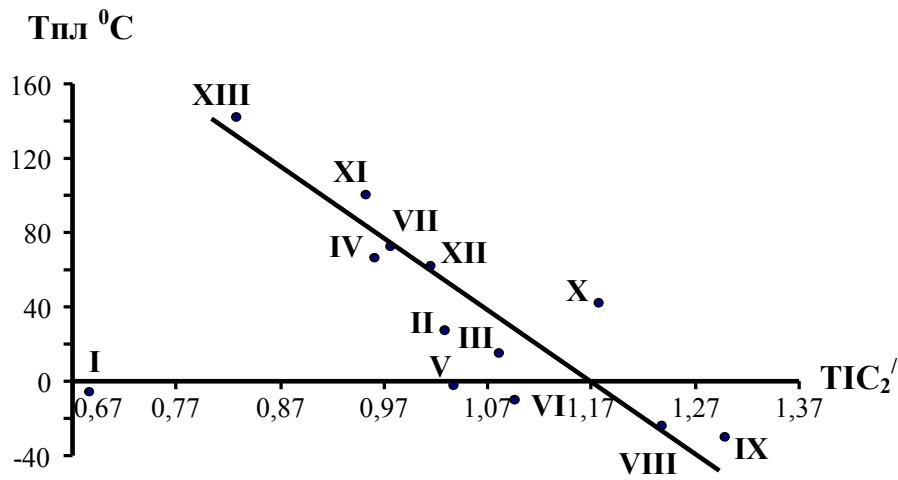

Рис. 5. Корреляционная зависимость $\mathrm{f}\left(\mathrm{TIC}_{2}{ }^{\prime}\right)-\mathrm{T}_{\text {пл }}$ для (I-XIII)

применить данную методику расчета индексов для прогнозирования свойств таких аддуктов для их дальнейшего направленного синтеза.

\section{Список литературы}

1. Урядов В.Г., Аристова Н.В., Офицеров Е.Н. Топологический подход к описанию влияния растворителя на реакционную способность аценов в реакции диенового синтеза. Бутлеровские сообщения. 2002. T.3(11). C. 7-12. [ Uryadov V.G., Aristova N.V., Ofitserov Y.N. Topological approach to the description of the effect of solvent on the reactivity of acenes in the reaction of diene synthesis. Butlerov Communications 2002. Vol. 3(11). P. 7-12. (In Russ.)]

2. Урядов В.Г., Офицеров Е.Н. К расчету коэффициентов пропорциональности в уравнениях взаимосвязи энергии активации реакции циклоприсоединения в растворе и энтальпии испарения растворителя. Бутлеровские сообщения. 2006. Т. 8(3). С. 14-19. [ Uryadov V.G., Ofitserov Y.N. About calculation of the proportionality coefficients in the equations of the relationship of energy of activation cycloaddition reactions in solution and the enthalpy of evaporation of the solvent. Butlerov Communications 2006. Vol. 8(3). P. 14-19. (In Russ.)] 
3. Урядов В.Г., Офицеров Е.Н. К вопросу активации реакций циклоприсоединения в растворе. Бутлеровские сообщения 2005, T.6(1). C.13-28. [ Uryadov V.G., Ofitserov Y.N. About the question of the activation of cycloaddition reactions in solution. Butlerov Communications. 2005. Vol.6(31),P.13-28. (In Russ.)]

4. Chemical applications of topology and graph theory. Under Ed. R. King. USA, 1987. $589 \mathrm{p}$.

5. Салахов М.С., Гречкина О., Багманов Б. Топологические индексы в корреляционном анализе реакции Дильса-Альдера. Россия, Palmarium Academic Publishing 2012. 94 c. [M.S. Salakhov, O.T. Grechkina, B.T. Bagmanov. Topological indices in correlation analysis of Diels-Alder reaction. Russia, Academic Publishing 2012. 94 p. (In Russ.)]

6. Salakhov M.S., Grechkina O.T., Bagmanov B.T. Topological approach in determining the structure-property dependence in a series of 4-cyclohexene-1,2-dicarboxylic acids. Journal of Structural Chemistry. 2009. Vol. 50(1). P. 179-183.

7. Salakhov M.S., Grechkina O.T., Bagmanov B.T. Application of topological indices in correlation analysis of N-alkylcarboxyl-imides of cyclic 1,2-dicarbonic acids. Journal of Structural Chemistry. 2010. Vol. 51(1). P. $16-22$.

8. Salakhov M.S., Grechkina O.T., Bagmanov B.T. Correlation prediction of acidity and melting point of diene adducts of hexachlorcyclopentadiene witch $\mathrm{N}$-alkylcarboxyimides of maleic, cyclohexene-, and endic 1,2-dicaboxylic acids. Journal of Structural Chemistr. 2010. Vol. 51(5). P. $847-853$.

9. Салахов М.С., Гречкина О.Т., Багманов Б.Т. Направленный синтез диеновых аддуктов 1,2,3,4-тетрахлор-5,5-диалкоксициклопентадиена с ангидридами 4-циклогексен-1,2дикарбоновых кислот на основе зависимости структура-свойство. Журнал Сибирского федерального университета. 2012. T.5(4). C. 343-351. [M.S. Salakhov, O.T. Grechkina, B.T. Bagmanov. Directed synthesis of diene adducts of 1,2,3,4-tetrachlor-5,5-dialkoxycyclopentadiene with anhydrides of 4-cyclohexene-1,2-dicarboxylic acids on the basis of dependence of structure-property. Journal of Siberian Federal University. Chemistry 2012. V.5(4). P. 343-351. (In Russ.)]

10. Салахов М.С., Гречкина О.Т., Багманов Б.Т., Умаева В.А. Направленный синтез $\mathrm{N}$-алкилкарбоксиимидов 1,4,5,6,7,7- гексахлорбицикло /2,2,1/ гепт - 5-ен - 2, 3- дикарбоновой кислоты на основе корреляционного анализа структура-свойство. Доклады АН Азербайджана. 2011. T.57(2). C. 74-81. [M.S. Salakhov, O.T. Grechkina, B.T.Bagmanov, V.S. Umaeva. Directed synthesis of N-alkyl carboxamides 1,4,5,6,7,7- hexachloro bicyclo / 2.2.1 / hept - 5-ene-2,3 dicarboxylic acid based on correlation analysis of structure-property. Report of the National Academy of Sciences of Azerbaijan 2011. Vol. 57(2). P. 74-81. (In Russ.)]

11. Салахов М.С., Гречкина О.Т., Багманов Б.Т., Умаева В.А. Синтез новых $\mathrm{N}$-алкилкарбоксиимдов эндо-бицикло /2.2.1/-гепт-5-ен-2,3-дикарбоновой кислоты с свойствами, прогнозированными на основе анализа зависимости «топологический индекссвойство». Доклады АН Азербайджана. 2011. T.57(5). C.88-93. [M.S. Salakhov, O.T. Grechkina, B.T. Bagmanov, V.S. Umaeva. Sinthesis of new N-alkylcarboxyimides of endo-bitciklo/2.2.1/hept-5-en-2,3- dicarboxylic acid with properties forecasted on the basis of analysis of dependence "topological index-property". Report of the National Academy of Sciences of Azerbaijan 2011. Vol. 57(5), P.88-93. (In Russ.)]

$$
-567-
$$


12. Stankevich M.I., Stankevich I.V., Zefirov N.S. Topological indices in organic chemistry. Russ. Chem. Rev. 1988. Vol. 57(3). P.191-208.

13. Schultz H.P, Schultz E.B., Schultz T.P. Topological organic chemistry. 2. Graph theory matrix determinants and eigenvalues and topological indices alcanes. J. of Chemical Information and Computer Sciences. 1990. Vol. 30(1). P.27-35.

14. Randic M. Haracterization of molecular branching. J. Am. Chem. Soc. 1975. Vol. 97(23). P.6609-6615.

15. Trofimov M.I., Smolenskii E.A. Application of the electro negativity indices of organic molecules to tasks of chemical informatics. Russian Chemical Bulletin. 2005. Vol. 54(9). P.22352246.

16. Зефиров Н.С., Кирпиченок М.А., Измайлов Ф.Ф., Трофимов М.И. Схемы расчета электроотрицательности атомов в молекуле в рамках принципа Сандерсона. Доклады AH CCCP. 1987. T. 296. C.883-887. [N.S. Zefirov, M.A. Kirpichenok, F.F. Izmaylov, M.I. Trofimov. Schemes for calculating the electronegativity of the atoms in the molecule under the principle of Sanderson. Reports of the USSR AS, 1987. Vol.296. P.883-887. (In Russ.)]

17. Кирпиченок М.А., М Трофимов.И., Зефиров Н.С. Доклады АН СССР. Сер. Хим. наук. 1989. T.304. C.887-896. [M.A. Kirpichenok, M.I. Trofimov, N.S. Zefirov, Reports of the USSR AS, 1989, Vol.304, P.887-896. )In Russ.)]

18. Balaban A.T. Distance connectivity index. Chem. Phys. Lett. 1982. Vol.89. P.399-405.

19. Салахов М.С., Гречкина О.Т., Багманов Б.Т. и др. Модифицированные теоретикоинформационные индексы органических молекул в решении задачи «структура-свойство». Сб. материалов научно-практической конференции «Актуальные проблемы современной биологии и химии». Гянджа, 2014. C. 45.[M.S. Salakhov, O.T. Grechkina, B.T. Bagmanov, Z.T. Abbasov. Modified theoretical information indices of organic molecules for the solution of the problem "structureproperty". Scientific-practical conference "Actual problems of modern biology and chemistry “. Ganja, 2014. P. 45.(In Russ.)]

20. Салахов М.С., Гречкина О.Т., Багманов Б.Т., Аббасов 3.Т. Новый подход к методике расчета теоретико-информационных индексов органических молекул с учетом электроотрицательности и радиусов атомов. Сб. материалов V Международной научно-практической конференции «Научные перспективы XXI века. Достижения и перспективы нового столетия», Россия, Новосибирск. 2014. Т.5(5). C.150-154 [M.S. Salakhov, O.T. Grechkina, B.T. Bagmanov, Z.T. Abbasov. A new approach to the calculation procedure of information-theoretic indices of organic molecules based on the electronegativity and atomic radii. V International scientific and practical conference «Scientific perspectives XXI century. Achievements and prospects of the new century», Novosibirsk, Russia 2014. Vol. 5(5), P.150-154. (In Russ.)]

21. Салахов М.С., Гречкина О.Т., Багманов Б.Т. Перспективы применения модифицированных теоретико-информационных индексов в решении задачи «структура-свойство» бромированных имидов циклических дикарбоновых кислот. Сб. докладов VII Международной научно-практической конференции «Теоретические и прикладные аспекты современной науки», Россия, г. Белгород. 2014. C.57-63. [M.S. Salakhov, O.T. Grechkina, B.T. Bagmanov. Prospects for the use of the modified information-theoretic indices in the task of "structure-property" brominated imides of cyclic dicarboxylic acids. VII International scientific 
and practical conference "Theoretical and applied aspects of modern science", Russia, Belgorod 2014. P.57-63. (In Russ.)]

22. Bagmanov B.T. Effect of structural factors and solvent nature in bromination of anilines Russian Journal of Applied Chemistry 2009. Vol. 82(9). P.1570-1576.

23. Salakhov M.S., Bagmanov B.T., Umayeva V.S., Bagmanova M.I. Oxidative bromination of aniline its derivatives. Russian Journal of Applied Chemistry. 2008. Vol. 81(8). P.1479-1481.

24. Кривенько А.П., Астахова Л.Н. Реакции электорфильного замещения в аренах. Саратов: Научная книга, 2008. 54 с. [A.P. Krivenko, L.N. Astakhova. Reactions electrophilic substitution arenas. Saratov: Scientific book, 2008. 54 p. (In Russ.)] 